\title{
Long-term results of the Binkhorst iris clip lens in senile cataract
}

\author{
JOHN L. PEARGE \\ Redditch, England
}

The purpose of this paper is to consider the long-term results of the iris clip lens in senile aphakia. It is not intended to discuss the many different forms of intraocular lens implants. Ophthalmic surgeons are well aware of the discouraging short- and long-term failure rate when contact lenses are used to correct aphakia, and this has stimulated renewed interest in intraocular lens implants in both Europe and America. Bonnet, Massin, and Gerhard (1966), reviewing 308 adult unilateral aphakics, found that after Io years only 20 per cent. were still wearing their contact lens. Mills and Lewis (197I), reviewing I $3^{2}$ patients who had been fitted with scleral contact lenses between $196 \mathrm{r}$ and I 966 inclusive, found at the end of I 968 that 46 per cent. were no longer wearing their contact lenses, although nine had worn the contact lens successfully before cataract extraction on the second eye. Despite this, the authors concluded that the insertion of the intraocular lens as a primary procedure is not indicated in patients, who, at the time of preoperative assessment, seem suitable for contact lens fitting. Martin Walker (1968), in a 5 -year survey of 74 patients for whom contact lenses were authorized, found that only 3 I were still wearing their contact lens at the time of the review. It is difficult to convince a unilaterally aphakic patient, who has good vision in his phakic eye and is unable to tolerate a contact lens, that the operation that he has undergone is a success, even if he has useful visual function in the aphakic eye. The Figure shows the iris clip lens in position.

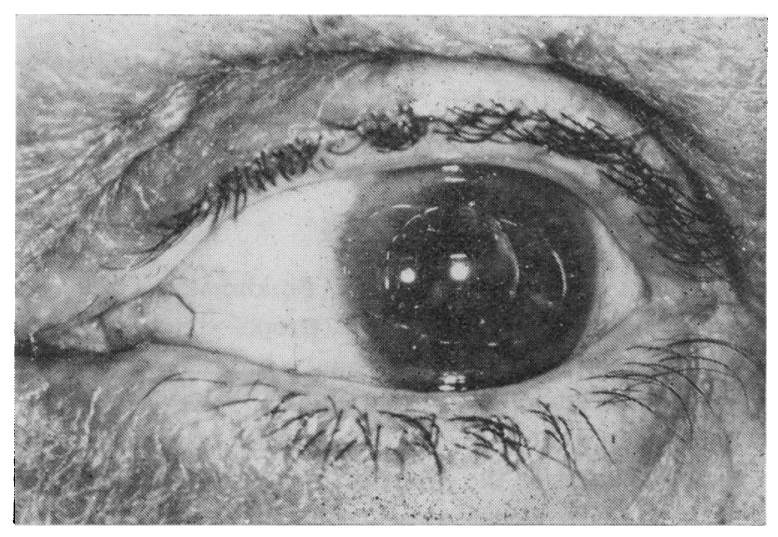

FIGURE Case 230 (see p. 325). Organized exudate on iris clip lens. 


\section{Advantages of iris clip lens implants in aphakia}

Inconvenience to the patient is minimal once the implant is satisfactorily placed. The patient has to accept only the regular instillation of topical pilocarpine to maintain miosis. He does not need to handle a contact lens, which so many patients, especially the elderly, find difficult if not impossible. Moreover the optical effect of an intraocular lens is better than that of a contact lens. Giraud, Friedman, Moore, Blau, Binkhorst, and Gobin (r g62) found that there was a higher degree of aniseikonia in monocular aphakia corrected with contact lenses than with intraocular lenses. The average difference in retinal image size was about 7 per cent. in the contact lens cases, and only about 2 per cent. in the iris clip lens cases. Binkhorst (1964) stated the average aniseikonia was $0 \cdot 2$ per cent. Giraud and others (1962) concluded that, while an intraocular implant offered a better opportunity for binocular vision in cases of monocular aphakia, they were worried about the danger of uveitis, glaucoma, and corneal dystrophy.

The advantage of the iris clip lens over the anterior chamber fixation lenses of Choyce, Strampelli, and Dannheim, is that the iris clip lens is situated further from the corneal endothelium, with no angle contact, thereby lessening the likelihood of the formation of peripheral anterior synechiae and endothelial dystrophy. It is also independent of the diameter of the anterior chamber. However, the technical problems of iris clip lens implantation are greater than those of the anterior chamber angle fixation lens, especially in cases requiring secondary implantations, in which a knuckle of vitreous may be present in the anterior chamber.

\section{Possible disadvantages of iris clip lenses}

Choyce (1959) suggested that the iris clip lens might produce pupil blockage and that, in time, the loops of the lens might lose their resilience and come adrift. Ridley (1959) doubted that the iris-supported lens would remain stable. Schweitzer (1959) suggested that the iris sphincter might atrophy. All these comments were suggestions of possible disadvantages.

Other workers have warned of the danger of late corneal dystrophy, and of the difficulty of treating a subsequent aphakic retinal detachment. In such cases dilatation of the pupil to provide adequate visualization of the retinal periphery might prove impossible.

There is still much doubt as to the advisability of employing intraocular implants. Barraquer (1959) stated that he had had excellent results with 4 I I anterior chamber Dannheim type lenses, but he later stated that because of various complications he had had to remove this type of lens in 200 out of 400 cases (Barraquer, 1970).

In the light of these doubts it was felt that an independent report on the long-term effects of iris clip lenses in aphakia would be valuable. Dr. Binkhorst was approached by the author and agreed to an independent examination of his cases.

\section{Selection of cases}

A list of over 600 cases was presented to the author by Dr. Binkhorst. These were numbered consecutively, the oldest implant being number one. It was proposed to examine only cases of elective intracapsular senile cataract extractions in which primary implantation of the iris clip lens had been effected, thereby avoiding all cases of traumatic cataract, previous glaucoma surgery, secondary iris clip lens implantations, or any other previous intraocular surgery. The rationale for this method of selection was to exclude changes in the eye which could have been caused by surgery, so that some comparison could be made between intracapsular cataract extraction with a lens 
implant and simple intracapsular extraction. Secondary lens implantation cases were excluded because a second incision into the anterior chamber could cause such complications as corneal dystrophy and peripheral anterior synechiae.

A suitable consecutive number of cases (from $3^{8}$ to 406 ) complying with these requirements was selected, and a letter was written to the last known address, to ask the patients to attend for examination. Of 124 patients approached, 27 had died and a further 25 were unable to attend for a variety of reasons. Excluding the deaths, 74 per cent. of those approached were examined.

\section{Method of examination}

All these patients who had been operated on by Dr. Binkhorst were examined by the author. Investigations included:

(x) Visual acuity with and without correcting spectacles; also objective refraction.

(2) Examination of the anterior segment of the eye with the Haag-Streit 900 slit-lamp microscope; applanation tonometry; gonioscopy using the Goldmann single-mirror lens.

(3) Fundoscopy with the direct ophthalmoscope; and in certain cases with the Keeler indirect ophthalmoscope.

(4) Goldmann visual fields in selected cases.

(5) Orthoptic report in suitable cases.

\section{Surgical technique}

It is not proposed to give a detailed description of the surgical technique, as this has been adequately described (Binkhorst and Leonard, 1968). Dr. Binkhorst's current method includes the following procedures:

Operation under general anaesthesia using muscle relaxants.

Limbal-based conjunctival flap, with keratome and scissors.

Corneo-scleral incision with one pre-placed Perlon suture.

Two or three peripheral iridotomies.

Capsule forceps extraction of the lens, preferably without the use of alpha-chymotrypsin.

Implantation of the iris clip lens using special introductory forceps in the horizontal meridian (Dr. Binkhorst has now changed to introduction in the vertical meridian.)

Instillation of acetylcholine into the anterior chamber to constrict the pupil onto the lens.

Tying of the pre-placed suture and filling of the anterior chamber with air.

Sealing of the corneo-scleral wound with a further eight or nine post-placed corneo-scleral sutures of Perlon.

Extraction of air from the anterior chamber so as to leave the iris at the level of the anterior chamber angle.

Suturing of conjunctival flap with interrupted sutures.

Subconjunctival injection of $0.5 \mathrm{ml}$. Depomedrone.

Application of eserine ointment into the lower fornix, and covering of the eye with a cartella shield.

Postoperative instillation of steroid with antibiotic drops four times a day and pilocarpine drops twice a day.

In the past few years some modifications in technique have taken place, notably the use of monofilament Perlon instead of 8-o virgin silk to close the corneo-scleral wound.

\section{Case distribution}

72 eyes were examined ( 38 right and 34 left). The average age was 73.9 years at the time of examination (range 43 to 96 ). The average time since the primary implantation of the iris clip 
lens was 4 years and 3 months (range 9 months to ro years). Thirty cases were unilateral implantations, the rest bilateral.

\section{Operative complications}

The notes of all cases were discussed by the author with Dr. Binkhorst and the following operative complications were noted:

(a) Lens capsule rupture occurred in three cases ( $116,230,370$ ), none of which had a poor visual result due to capsular or cortical remnants.

(b) Vitreous presentation in the wound occurred in one case (352), but there was no actual vitreous loss. This patient has a visual acuity of $6 / 9$ corrected 20 months after surgery. Gonioscopy shows a small incarceration of vitreous in the wound.

Binkhorst and Leonard ( 1967$)$ noted that the incidence of vitreous presentation in secondary implantations is often unavoidable. It occurred in eleven out of seventy aphakic eyes (an incidence of 16 per cent.), the inference being that primary implantation of the iris clip lens is less hazardous to the vitreous body than secondary implantation after intracapsular cataract extraction.

\section{Postoperative complications}

\section{SHALLOW ANTERIOR GHAMBER}

This occurred in four cases, an incidence of just over 5 per cent. Giardini and Pagliaga (1964), using keratome and scissors or a von Graefe section with post-placed corneoscleral sutures, found that when they used five to six corneo-scleral sutures $14^{\cdot 8}$ per cent. of cases had flat anterior chambers and 6.2 per cent. had delayed re-formation of the anterior chamber. However, with eleven or more corneo-scleral sutures, only 3 per cent. had flat anterior chambers and I 6 per cent. had delayed re-formation of the anterior chamber after cataract surgery. However, Chandra, Singh, and Sharma (1962), using four or more post-placed corneo-scleral sutures in cataract surgery, found an incidence of $i \mathrm{I} \cdot 6$ per cent. of shallow anterior chambers.

An examination of the literature shows that the incidence of shallow anterior chamber after cataract surgery varies from nil to 25 per cent. of cases, depending on the type of section and the number of corneo-scleral sutures used.

Case 97 Shallow anterior chamber was associated with postoperative iris prolapse, which required subsequent surgical intervention. Nearly 8 years after implantation the visual acuity could be corrected to 6/24. There had been a minimal amount of epithelial corneal oedema in the upper limbal area with a healthy endothelium. Macular changes, which are present, may account for some of the reduction in visual function. There is also 30 per cent. peripheral anterior synechiae to the corneal wound.

Case 186 Shallow anterior chamber developed 3 months after surgery. There is now present a slight Fuchs's corneal dystrophy with 80 per cent. peripheral anterior synechiae to Schwalbe's line, but the visual acuity can be corrected to $6 / 95$ years after implanation.

Case 290 A shallow anterior chamber was present for 6 weeks. There are no peripheral anterior synechiae. The visual acuity is $6 / 18$ some 2 years and 7 months after implantation.

Case 368 A shallow anterior chamber was present for 6 days. There is io per cent. peripheral anterior synechiae to the trabeculae, and the corrected visual acuity is $6 / 66$ months after surgery.

RETINAL DETACHMENT

This occurred in one case (347) in this series, an incidence of $1 \cdot 3$ per cent. Duke-Elder ( 1969 ) stated that retinal detachment may be expected in 2 per cent. of cases after extra- 
or intracapsular cataract extraction. The detachment occurred 4 months after the original surgery; it was successfully treated with diathermy and the corrected visual acuity is now 6/12. In this series, the incidence of aphakic retinal detachment is no higher than that expected in routine intracapsular cataract extractions. In fact, it can be postulated that an iris clip lens can give increased stability to the aphakic vitreous body by replacing part of the support afforded by the crystalline lens, and thereby reducing traction on the vitreo-retinal adhesions, and may, therefore, reduce the likelihood of retinal detachment in the aphakic eye.

\section{STRIATE KERATITIS}

This condition is commonly noted after routine cataract extraction without artificial lens implantation. Marked striate keratitis was noted in the following four cases:

Case 53 The striate keratitis cleared, giving a corrected visual acuity of 6/6. Subsequently the vision has deteriorated to $6 / 36$, because of senile macular degeneration, the patient being 96 years of age.

Case 64 The striate keratitis progressed to bullous keratopathy, the visual acuity being counting fingers only. It may have been that there was corneal endothelial damage at the time of operation, because of the use of intracameral epinephrine. This practice has now been abandoned.

Case II 3 Striate keratitis was present for the first postoperative week. It was probably caused by a tear in Descemet's membrane, which occurred during manipulation of the iris clip lens at operation. However, the corrected visual acuity is now 6/12.

Case 364 The cornea is now clear with a corrected visual acuity of $6 / 9$.

\section{MAGULAR OEDEMA}

This condition is an accepted complication in aphakia. Duke-Elder (1969) suggested that macular oedema occurred in 2 per cent. of cases after uncomplicated cataract extraction. The onset may be rapid, developing to a macular hole. However, there are cases in which the oedema subsides with the return of some visual function.

It has been suggested that several factors are associated with aphakic macular oedema, such as ultraviolet radiation, vascular instability, and rupture of the anterior hyaloid membrane (Gartner, I965). Binkhorst and Leonard (1967) reported that macular disorders responsible for a visual acuity of 6/1 2 or less occurred in fifteen out of seventy secondary lens implantation cases, and in seventeen out of 124 primary implantations of iris clip lenses, an incidence of 12 per cent.

Case $3^{8}$ The visual acuity deteriorated to $6 / 24$, but has now improved to $6 / 12$.

Case 923 months after surgery the corrected visual acuity was $6 / 9$, but then deteriorated to $6 / 18$, at which level it still remains.

Case 329 Postoperatively the visual acuity was $6 / 9$, macular oedema then reduced it to $6 / 24$, but it has subsequently improved to its present level of $6 / 9$ corrected.

Case ${ }_{364}$ Macular oedema developed but subsided, leaving a visual acuity, when examined, of $6 / 9$ with a normal fundus picture.

Case 370 Macular oedema is still present on examination, and the visual acuity is $6 / 36$ corrected.

These five cases represent an incidence of 5 per cent. postoperative macular oedema, but of these three showed improved visual function, with the disappearance of the oedema. 
SERIOUS CORNEAL GOMPLIGATIONS

Bullous keratopathy is an infrequent sequela of routine cataract extraction without artificial lens implantation, the incidence being usually less than I per cent. It was noted in the following cases:

Case 64 During the operation epinephrine was instilled into the anterior chamber. The iris clip lens dislocated, and required manipulation in order to reposition it within the pupil. Intense striate keratitis was noted postoperatively with persistent corneal oedema for some 2 months. The visual acuity improved to $6 / 9$ corrected, but then deteriorated to its present level of counting fingers. It would seem likely that the epinephrine solution caused corneal endothelial damage at the time of operation.

Case 139 Primary implantation was carried out $6 \frac{1}{2}$ years ago. The visual result was $6 / 12$, but the present acuity is only hand movements, because of bullous keratopathy in this 85 -year-old patient. There is no corneal endothelial contact with the loops or optical part of the lens.

These two cases represent an incidence of 2.7 per cent. in the examined series. It is probable that the corneal endothelium was damaged at surgery in the first case by manipulation of the lens and the use of intracameral epinephrine. However, there was no obvious surgical reason for the second case of keratopathy.

\section{LESS SERIOUS CORNEAL GOMPLICATIONS}

Case 97 Epithelial corneal oedema in the upper one-third with healthy endothelium. The patient had suffered an iris prolapse and flat anterior chamber after surgery. The visual acuity is now 6/24 corrected.

Case I $_{3}$ This case has already been mentioned in the paragraph concerning striate keratitis; the iris clip lens having dislocated postoperatively, it required repositioning with a needle knife, and this resulted in a tear in Descemet's membrane, the visual acuity now being 6/1 2 .

Case 154 This woman had noticed deterioration of vision some 12 months previously. She had not used her pilocarpine drops regularly. The visual acuity was counting fingers. There was corneal oedema in the temporal half because of contact from an anterior dislocated lens. The lens was successfully manipulated into the pupil after dilatation. The cornea has since cleared, but the visual acuity remains low because of macular oedema. (This case was not included in those with macular oedema, as this complication was reported to the author some months after the original examination of the patient.)

Cases $6_{3}$ and 186 This is a patient of 81 with bilateral Fuchs's corneal dystrophy and senile macular degeneration. The visual acuity in the right eye is less than $6 / 60$, but that in the left is $6 / 9$.

Case 384 Fuchs's corneal dystrophy with macular changes and a visual acuity of $6 / 36$.

Case 373 This is a diabetic patient with macular changes; there is minimal epithelial corneal oedema and the visual acuity is less than 6/60.

Fine dusting of iris pigment on the corneal endothelium occurred in 28 out of the 72 examined cases. This finding is frequently noticed after any anterior segment surgery. It has virtually no effect on the visual acuity and is not a complication peculiar to iris clip lens implantation.

ANTERIOR UVEITIS

Evidence of old or active uveitis was looked for during slit-lamp microscopy, as gradual breakdown of the acrylic lens and the supramid loops could irritate the iris and ciliary body. Old uveitis was detected in the following cases: 
Case 74 There was postoperative hyphaema with a low-grade iritis for 6 months. There are tuo filamentary posterior synechiae to the vitreous face. The eye is quiet with a visual acuity of $6 / 9$ and it is likely that the pupil could be dilated.

Cases 230 and $37 \mathrm{I}$ This is a bilateral case, in which there is an organized exudate partly covering the lens of the implant in both eyes. The first eye suffered a ruptured capsule during surgery, and epinephrine was instilled into the anterior chamber. The visual acuity in this eye is now $6 / 6$ corrected (Figure I). The other eye has a similar but small exudate on the lens with a corrected visual acuity of $6 / 18$. Both eyes are now quiet with a clear aqueous.

Case 374 There are small old precipitates on the implant, not affecting vision, but there are also macular changes with a visual acuity of $6 / 36$.

Case 383 There is a small patch of old precipitate on the iris clip lens, not affecting the vision. The eye is quiet and the visual acuity is $6 / 6$.

Fifteen cases were detected with one or two white cells in the anterior chamber. None of these had any protein flare. Three of the patients had had the artificial lens implant for more than 5 years, the other twelve less than 5 years. Detection of the occasional white cell in the aqueous is not uncommon in the anterior chambers of aphakic eyes without artificial lens implants, and does not seem to be of any great significance.

\section{IRIS ATROPHY}

Schweitzer ( I959) suggested that there might be a danger of atrophy of the iris sphincter, if an iris clip lens is used. However, this was not found in any of the cases examined. Some loss of iris pigment had occurred in 37 cases in relation to the posterior loops of the lenses. Of these 37 patients, fifteen had had their implants for more than 5 years and 22 for less. Pigment loss in relation to the supramid loops of the lens can occur from operative manipulation or subsequently from rubbing against the iris. In any event, in no case had the posterior loop pushed through the iris, so that this would seem to be of minimal importance.

\section{POSTOPERATIVE DISLOCATION OF THE IRIS CLIP LENS}

Unless there are sufficient adhesions between the pupillary margin and the posterior loops of the iris clip lens to ensure stability, it is advisable to use a miotic such as pilocarpine for some considerable time postoperatively to maintain constriction of the sphincter on the posterior loops. Of the 72 patients examined, eight (I I per cent.) had suffered at least one dislocation of the lens. Of the thirteen actual dislocations, ten lenses had been repositioned by manipulation alone, by dilating the pupil and rotating the patient until the lens is in situ in the pupil, and then constricting it with a miotic. The other three cases had needed repositioning with the use of a needle knife in the anterior chamber. The average age of the patients suffering dislocation was 63.5 years, and that of the whole series was 73.9 years. It would thus seem that the younger patients are more prone to dislocation of the lens.

Case 56 Five dislocations had occurred in the 9 years since the original implantation had been made. Repositioning was effected by manipulation. Finally, the eye was opened and the lens was sutured to the iris with perlon, one stitch being used on each anterior loop. The corrected visual acuity is now 6/9, and there have been no subsequent dislocations of the iris clip lens. This patient persistently failed to instil pilocarpine drops. 
Case 113 The lens dislocated 2 years after surgery. It was repositioned by manipulation, and the visual acuity then returned to $6 / 12$. A further dislocation required the use of a needle knife in the anterior chamber. The visual acuity is now 6/12 corrected.

Case ${ }_{2} 8$ The lens dislocated 3 months after surgery and was repositioned with a needle knife in the anterior chamber. Corneal oedema persisted for 2 years, but this cleared and the corrected visual acuity is now $6 / 6$.

Case 130 The lens dislocated 4 months after surgery and was manipulated into position. The cornea is clear and the visual acuity is $6 / 5$ corrected.

Case I4I The lens was manipulated back into position. The cornea is clear with a visual acuity of $6 / 6$ corrected.

Case I53 The lens dislocated 3 years after surgery, and was repositioned with a needle knife. The cornea is clear and the visual acuity is $6 / 9$ corrected.

Case ${ }_{154}$ This is the patient already described, who attended this investigation with corneal oedema due to anterior dislocation of the lens. The lens was manipulated into position and the corneal oedema has subsequently cleared completely, but the visual acuity remained poor because of macular oedema. The patient had not been using miotics.

Case 395 The iris clip lens dislocated in the immediate postoperative period and was manipulated back into position. The cornea is now clear with a visual acuity of $6 / 6$.

Binkhorst (1962) stated that the prolonged use of miotics was not necessary. However, Binkhorst and Leonard ( 1967 ) noted dislocation of the iris clip lens in 24 out of 208 cases, and concluded that miotics were, therefore, advisable. Dallas (1970), reviewing 92 patients with iris clip lens, had three cases of early postoperative dislocation of the lens, one lens being subsequently removed. He has had no late dislocations and keeps his patients on pilocarpine 2 per cent. indefinitely. Jardine (1970) suggested that the Federov design of clip lens may be less liable to dislocation. It should be remembered that most of the patients who suffered dislocation in Binkhorst's series with corneal oedema regained very useful vision with a clearing of the cornea after repositioning of the lens. It would seem, however, that, unless there are adequate posterior synechiae to the posterior loops of the iris clip lens, or the lens is sutured to the iris, the prolonged use of miotics is indeed mandatory, especially in the younger patient. It is for this reason that, in my own series of cases, a perlon iris stitch is placed to fix an anterior loop of the iris clip lens. Worst (197I) similarly sutures his iris clip lenses to the iris with perlon.

Dilatation OF THE PUPIL

The margins of the pupil were critically examined under the slit-lamp microscope for evidence of adhesions between the pupillary margin and the posterior loops of the lens and the anterior vitreous face, to assess whether the pupil could be dilated for full peripheral fundoscopy. Only eight pupils of the 72 cases would probably not dilate if necessary although full dilatation would carry the risk of lens dislocation. A frequent criticism of iris clip lenses is that the examination of a case of aphakic retinal detachment with the indirect ophthalmoscope would be difficult. However, it will be remembered that the pupil in an iris clip lens is approximately $4 \mathrm{~mm}$. square. It will not constrict further however strong the light stimulus. Also, providing one is not using a strong mydriatic, the pupil could be dilated to $8 \mathrm{~mm}$. without the lens dislocating, because of the size of the anterior and posterior loops. In examining a proportion of the patients, indirect ophthalmoscopy was carried out with little difficulty. 
FUNDOSGOPY

Direct, and in some cases, indirect ophthalmoscopy was performed. No retinal detachments were found. Macular oedema or macular degeneration was found in fourteen ( 19 per cent.) of a series of patients whose average age was 73.9 years. The average age of the patients with maculopathy was 7 I years. Macular lesions may well have been present before surgery. It has long been noted that there is a high incidence of macular lesions after uneventful cataract surgery; the lesion may be initiated by the cataract surgery, possibly because of postoperative hypotension or surgical uveitis (Gillman, I965).

INTRAOGULAR PRESSURE

Applanation tonometry was performed with a Goldmann tonometer. The average intraocular pressure of the 72 cases was $16 \mathrm{~mm}$. $\mathrm{Hg}$ (range io to 24). In only one case was the intraocular pressure $24 \mathrm{~mm}$. $\mathrm{Hg}$. There was no other evidence of glaucoma in this series of cases.

PATHOLOGICAL GHANGES IN THE ANGLE OF THE ANTERIOR GHAMBER

The drainage angles in all cases were examined with a single mirror Goldmann gonioprism. Fourteen patients were noted to have peripheral anterior synechiae (PAS). One case with 80 per cent. PAS had a flat anterior chamber in the postoperative period, but the intraocular pressure when noted was normal. In this I9 per cent. of cases with PAS, the average amount of PAS in the angle was I 7 per cent. In my own series ( I 968) of I I 5 intracapsular lens extractions, 35 per cent. of cases had some degree of PAS. In addition, three of the 72 cases of the present series had a slight degree of angle recession not associated with glaucoma. The relatively low incidence of PAS in this series of cases is probably due to two factors:

(I) The centration of the iris clip lens tends to pull out any tags of iris in the wound;

(2) Filling the anterior chamber with air after, or even before, the first stitch has been tied.

It is also possible that cutting iridotomies instead of iridectomies lessens the incidence of iris tags incarcerated in the corneo-scleral wound.

It can be concluded that the iris clip lens has no visible effect on the angle of the anterior chamber.

CHANGES IN THE IRIS CLIP LENS

The lenses were examined carefully with the slit beam for any evidence of disintegration of either the acrylic of the lens or the supramid loops. The oldest lenses in situ appeared to be similar to those that had been implanted recently, and no evidence of disintegration of the plastic material of the lens implant was found.

VISUAL ACUITY

This was tested with Snellen's charts, both with and without spectacle correction. When necessary a pinhole lens was used or refraction was carried out.

Unaided visual acuity

The average unaided visual acuity was 6/24 (including only cases with a visual acuity of $6 / 60$ or better). 
Aided visual acuity (with spectacles or with a pinhole lens)

The average aided visual acuity was $6 / 12 ; 48$ cases $(67$ per cent.) had a visual acuity of $6 / 9$ or better with or without correction.

Only two patients had no useful vision. One had finger counting and the other hand movements, and both had bullous keratopathy. There was also significant reduction in visual function in all cases with macular changes. Binkhorst and Leonard (1967) found that, of 124 eyes with primary implantation of iris clip lens, 66 per cent. had a visual acuity of $6 / 9$ or better. In the present series, 23 cases $(72$ per cent. of the 32 with implants in situ for 5 years or more) had a visual acuity of $6 / 9$ or better, so that there would not appear to be any marked falling off of visual function with the passage of time.

\section{PHOTOPHOBIA}

23 patients (3I per cent.) admitted this symptom, but it was usually of slight degree and easily overcome by the use of tinted spectacles.

Some patients also noted lens movement on movement of the head, but this was not a severe complaint.

\section{BINOGULAR FUNCTION}

Orthoptic assessment was carried out in all cases, and it was found that patients with reasonable vision in both eyes had a slight to moderate degree of exophoria, more marked for near than for distance. Voluntary convergence was generally quite good. 53 patients (72 per cent.) had some degree of stereopsis when measured with the polaroid fly test and the Wirt rings. Girard and others ( 1962 ) concluded that intraocular lenses gave a better chance of binocular function than aphakic contact lenses. Of the thirty unilateral aphakics, 23 had useful vision in the aphakic eye, all of these had some degree of stereopsis and there was no complaint of diplopia.

VISUAL FIELDS

The effective visual field with a correcting spectacle lenticular lens is in the region of $30^{\circ}$, increasing with an aspheric lens to a $60^{\circ}$ field. There is also the factor of distortion in the mid-periphery of the visual field (Linksz, I965) that is experienced by an aphakic when wearing a normal spectacle correction. Goldmann perimetry was performed on eighteen selected cases, using various target sizes and light intensities.

\section{Using target size 3 and intensity 4}

The average temporal field in the horizontal meridian was $5^{\circ}$, and the nasal field $33^{\circ}$.

\section{Using target size 5 and intensity 4}

The average temporal field in the horizontal meridian was $69^{\circ}$, and the nasal field $39^{\circ}$.

The field of vision of an eye with an iris clip lens shows none of the defects in peripheral vision associated with a spectacle correction. That is, there is no area of uncorrected vision and no ring scotoma.

\section{Discussion}

It has been the intention of this paper to establish the advantages and disadvantages associated with the long-term implantation of the iris clip lens in aphakia. 
Complications peculiar to iris clip lens implants

Manipulation of an iris clip lens or other anterior chamber implant within the anterior chamber during surgery can cause corneal endothelial damage. In addition, endothelial damage can also occur by contact of the implant, or if the lens subsequently dislocates anteriorly. Serious bullous keratopathy occurred in two cases in this series; one may have been due to intracameral epinephrine at the time of operation, and the other may have also been due to some other operative misadventure. Dallas (1970) had four cases of corneal dystrophy in his series. The corneal oedema which patients suffered after anterior dislocation of the iris clip lens was reversible after the lens had been repositioned into the pupil. Dislocation of the iris clip lens is the main problem of long-term implantation. Patients, especially when infirm and living alone, may forget or be unable properly to instil the miotic drops. My own series of over roo cases have had fixation of one anterior loop of the lens with a Perlon iris stitch, and this has undoubtedly decreased the number of dislocations, none of which has required intraocular manipulation to reposition the lens. It has also obviated the need for miotics in most of the patients, but these are still required in some of the younger patients with active pupils, and also in those patients who have been conscious of slight shaking of the lens during ocular movements. (An operative point to remember is the importance of constricting the pupil after removal of the cataract, to ensure that the pupil will constrict over the loops of the iris clip lens-Binkhorst (I97I) has also made this point). The use of a perlon or platinumiridium iris stitch allows the pupil to be safely dilated to at least $8 \mathrm{~mm}$. Worst (I97 I) has similarly noted the value of the iris stitch in his series.

The incidence of late macular lesions deserves further investigation, but it must be remembered that some cases of macular oedema resolve with return of visual function. Lurie (197I) reported an incidence of about 5 per cent. of macular lesions in his series of cases.

\section{Long-term advantages of the iris clip lens}

Most important is the general patient satisfaction with the visual results of their operation, and in this series 87 per cent. of patients had enjoyed useful vision for over 5 years. Generally, binocular function is very good, as are the visual fields, and the patient is able to lead a much less inhibited life, than if aphakic spectacles have to be worn. Nor must the high rate of failure of contact lenses in aphakia be forgotten. Patients who have undergone surgery for a unilateral cataract, and are unable to tolerate a contact lens, consider the operation to have been a functional failure. Secondary implantations after contact lens failure carry the extra risk of another anaesthetic and of injury to the corneal endthelium, and a higher risk of operative vitreous loss (Binkhorst and Leonard, 1967).

This investigation has shown that the iris clip lens does not appreciably disintegrate over Io years, that the iris sphincter does not appreciably atrophy through irritation from the implant, that late uveitis is no problem, and that there is no appreciable damage to the drainage angle. Pupil block glaucoma did not occur in this series, but it can occur if the peripheral iridectomies or iridotomies are non-patent or are inadequate to maintain a satisfactory drainage of aqueous from the posterior to the anterior chamber. In view of this, I feel that two iridectomies are preferable to iridotomies (the pupil block if it occurs is easily overcome by slight dilatation of the pupil).

The corneal endothelium in the majority of patients is able to tolerate the presence of an iris clip lens implant for over 5 years. There is no evidence from this series of any increased incidence of aphakic retinal detachment. In fact, the virtual absence of a 
vitreous knuckle in the anterior chamber reduces the danger of vitreo-corneal adhesions which can produce an endothelial dystrophy, and it seems likely that the iris clip lens replaces some of the support afforded by the crystalline lens in the normal eye. In my own series of cases (Pearce, I968) 63 per cent. of 179 eyes after intracapsular cataract extraction had intact anterior hyaloids herniating into the anterior chamber. Hilding (1954) has listed the pathological potential of the altered state of the vitreous body in the aphakic eye as:

(a) Loss of the dampening effect on vitreous mobility by the loss of the crystalline lens;

(b) Increased antero-posterior dimension of the vitreous;

(c) Rupture of the anterior hyaloid with vitreo-corneal and vitreo-iris adhesions;

(d) Development of lines in the zones of tension;

(e) Separation of the vitreous into divided masses;

(f) Increased inertial stress in postrotational forces.

The implications of the above factors are stated by Irvine (1957) as:

(a) Additional stress on posterior vitreous attachments;

(b) Increased vitreous motion;

(c) Pull on the vitreo-retinal attachments at the macula, disc, and equatorial zone;

(d) Anterior displacement of the vitreous into an iris-contacting position as a basis of pupillary block glaucoma.

Gillman (1965) has suggested that 25 per cent. of aphakic detachments occur within 3 months of surgery, and 50 per cent. within one year. Dallas (1970) reported a 5-year follow-up of 92 iris clip lens cases, and these as well as my own series of over Ioo cases have so far been free from the complication of retinal detachment; it is therefore possible $\stackrel{\mathbb{D}}{\mathrm{D}}$ that a large follow-up of iris clip lens cases may show a reduction in the incidence of retinal $\vec{\partial}$ detachment. It would seem correct that a surgeon should not embark upon the technique of artificial lens implantation until he has performed a sufficient number of routine intracapsular cataract extractions, and is fully conversant with his technique and his own incidence of complications, such as shallow anterior chamber and vitreous loss. Because of its somewhat specialized technique, implantation should not be performed on an occasional basis. There is undoubted value in the use of the operating microscope, especially if iris fixation of the implant is to be attempted, as it is only by meticulous attention to surgical detail, avoiding any undue manipulations within the anterior chamber, that the $\frac{\rho}{?}$ implantation of artificial lenses into the pupil can be relatively free from complications.

\section{Summary}

72 cases of elective intracapsular senile cataract extraction with primary implantation of a Binkhorst iris clip lens were reviewed. The lenses had been in situ for up to ro years. It is concluded that the long-term incidence of complications is low and that the technique merits active consideration by experienced surgeons, especially those concerned with the $\bar{\varnothing}$ surgical treatment of unilateral aphakia.

My thanks are due to Dr. Binkhorst for his kindness in allowing me freely to examine his cases, to his secretary for arranging the examination of the patients, to Dr. Carmin Bennoto for performing the Goldmann $\stackrel{?}{\mathbb{D}}$ perimetry, to Miss Jos Bierlaagh of the University Eye Clinic, Leiden, who carried out the orthoptic investigations and helped with language problems, and to Mr. M. J. Roper-Hall for his helpful criticisms of the paper. This investigation was supported by a Keeler Award. 


\section{References}

BarraQuer, J. (1959) Trans. ophthal. Soc. U.K., 79, 581

(1970) Ibid., 90, 731 (in discussion of Dallas)

BinKhorst, c. D. (1962) Brit. F. Ophthal., 46, 343

- (1964) Ophthalmologica (Basel), 148, 169

(I97I) Personal communication and LeONARd, P. A. м. (1967) Amer. F. Ophthal., 64, part 5, p. 947

(1968) In "Perspectives in Oph thalmology", ed. H. E. Henkes, pp. 195, 2 10, 212. Excerpta Medica Foundation, Amsterdam

BoNNET, R., MASSIN, M., and Gerhard, J. P. (1966) Rapport annuel in Bull. Soc. Ophtal. Fr. (numéro spécial), p. 173

GHANDra, P., SINGH, D., and sharma, s. L. (1962) "XIX Conc. Ophthal. India (New Delhi) 1962,

Acta", vol. I, pp. 766-783

CHOYGe, P. (1959) Trans. ophthal. Soc. U.K., 79, $5^{82}$

DALlas, N. L. (1970) Ibid., 90, 725

DUKE-ELDER, S. (1969) "System of Ophthalmology", vol. 11, pp. 280, 286. Kimpton, London GARTNER, s. (1965) In "Complications after Cataract Surgery", ed. F. Theodore, p. 324. Churchill, London

giardini, A., and pagliaga, G. P. (1964) Brit. J. Ophthal., 48, I33

gillman, A. M. (1965) In "Complications after Cataract Surgery, ed. F. Theodore, p. 553. Churchill,

London

GIRARD, L. J., FRIEDMAN, B., MOORE, C. D., BLAU, R. I., BinkHORST, C. D., and GOBIN, M. H. (I962)

Arch. Ophthal. (Chicago), 68, 762

HILDING, A. C. (1954) Ibid., 52, 699

IRVINE, S. R. (1957) In "Symposium on Diseases and Surgery of the Lens", ed. G. M. Haik, p. I66.

Mosby, St. Louis

JARdine, P. (1970) Personal communication

IıNskz, A. (1965) In “Complications in Cataract Surgery", ed. F. H. Theodore, p. 6ro.

Churchill, London

LURIE, L. (197 I) Personal communication

MILls, P. V., and LEWIS, E. м. т. (1971) Brit. F. Ophthal., 55, I I6

PEARCE, J. L. (1968) In "A Detailed Comparison of $28 \mathrm{I}$ Cases of Intracapsular Cataract Extraction", p. 139. Univ. Birmingham

RIDLEY, H. (1959) Trans. ophthal. Soc. U.K., 79, 582 (in discussion of Binkhorst)

SCHWEIZER (1959) Ibid., 79, 582 (in discussion of Binkhorst)

WALKER, w. MARTIN (1968) Quoted in Brit. orthopt. F., 25, 78

WORST, J. (I97I) Personal communication 